\title{
Band-edge polarized optical absorption in europium chalcogenides
}

\author{
A. B. Henriques, A. Wierts, and M. A. Manfrini \\ Instituto de Física, Universidade de São Paulo, Caixa Postal 66318, 05315-970 São Paulo, Brazil \\ G. Springholz \\ Institut für Halbleiter- und Festkörperphysik, Johannes Kepler Universität, A-4040 Linz, Austria \\ P. H. O. Rappl, E. Abramof, and A. Y. Ueta \\ LAS-INPE, Avenida dos Astronautas, 1758-Jd. Granja, 12227-010 São José dos Campos, Brazil
}

(Received 20 June 2005; published 31 October 2005)

\begin{abstract}
The band-edge optical absorption of EuTe and EuSe, grown by molecular beam epitaxy, was measured, using circularly polarized light, in the Faraday configuration, at $T=1.8 \mathrm{~K}$. In thin layers (less than $0.4 \mu \mathrm{m}$ ), a doublet of absorption lines could be observed, with a full width at half maximum of about $50 \mathrm{meV}$ in EuTe and $80 \mathrm{meV}$ in EuSe. The lines are separated by about $200 \mathrm{meV}$ in EuTe and $300 \mathrm{meV}$ in EuSe, and they show a strong magnetic circular dichroism. The oscillator strength for these lines increases by a factor of almost 20 when the antiferromagnetic order is changed to ferromagnetic by the application of an external magnetic field. Faraday rotation in the transparency gap shows a strongly nonlinear dependence on the intensity of the magnetic field. These results are interpreted in the framework of the model of electronic transitions between the localized ground $\left|4 f^{7}\left({ }^{8} S_{7 / 2}\right) M=-\frac{7}{2}\right\rangle$ and excited states comprised in $\left|4 f^{6}\left({ }^{7} F\right) J M_{J} ; X\right\rangle$ [where $X$ represents the $5 d\left(t_{2 g}\right)$ states], yielding good agreement with experiment. The strong enhancement of the oscillator strength with increasing magnetic field may be an indication of exciton formation due to the compression of the zero-field Bloch-like extended electronic wave function.
\end{abstract}

DOI: 10.1103/PhysRevB.72.155337

PACS number(s): 78.20.-e

\section{INTRODUCTION}

Europium chalcogenides display a very rich spectrum of magnetic properties, associated with the large spins in the $\mathrm{Eu}$ sites in the crystal structure, due to the half-filled $4 f$ orbital in the $\mathrm{Eu}$ atoms. Because of this, europium chalcogenides have attracted much interest, and today they are probably one of the most thoroughly studied magnetic semiconductors. Their use in practical applications has been largely discarded ${ }^{1}$ because they display magnetic order only at very low temperatures, however, strain has been employed to bring the transition temperatures up in heteroepitaxial multilayers. ${ }^{2}$

Although europium chalcogenides have already been intensely investigated, some aspects of their band-edge absorption spectrum have still not been fully clarified. Narrow features are theoretically predicted to be observed, both in the Kasuya exciton model, ${ }^{3}$ or in the $d$-conduction band model, ${ }^{4}$ however, the direct observation of narrow features in their band-edge absorption spectra has not been reported yet, neither in bulk crystals nor in evaporated thin films, except for our preliminary report on thick epitaxial samples, in which a single sharp feature was seen in the EuTe absorption spectrum. ${ }^{5}$ A possible explanation is that direct absorption measurements are difficult because of the high absorption coefficient of single crystals, and the absorption spectra reported were deduced from the Fresnel ${ }^{6}$ or Kramers-Kronig analysis ${ }^{7}$ of the reflectivity spectra, which can be insensitive to sharp features; the absence of sharp features has also been ascribed to the presence of defects and lack of purity in the samples. ${ }^{6}$ Because of this, quantitative analysis of the band-edge absorption spectra has been largely limited to modeling of the Urbach tail (see, for instance, Ref. 16).
More recently, growth techniques, such as molecular beam epitaxy (MBE), have been used to produce thin epitaxial layers of very high purity and structural quality, and it has led to the discovery of a sharp photoluminescence (PL) line [full width at half maximum (FWHM) $10 \mathrm{meV}$ ] at $1.9 \mathrm{eV}$ in EuTe, which is described by a large effective $g$ factor, and shows a giant tuning with a magnetic field $(32 \mathrm{meV} / \mathrm{T}) \cdot{ }^{8-10}$ In this paper, we investigate the optical absorption edge of EuTe and EuSe grown by MBE. The spectra were measured using circularly polarized light, in the Faraday geometry. The samples had a thickness of a few tenths of a micron, which allowed us to measure the absorption edge up to photon energies of $2.5 \mathrm{eV}$. The small thickness of the samples also allowed us to avoid the strong Fabry-Perot interference fringes seen in the transmission spectra of thicker samples. ${ }^{10}$ When the ferromagnetic order is attained using an external magnetic field, a prominent doublet of narrow absorption lines is seen. The optical density of the lines increases with the thickness of the epitaxial layer, ${ }^{11}$ demonstrating that the absorption process occurs in the bulk of the epitaxial layer. The lines comprising the doublet have opposite circular polarizations, and are separated by about $200 \mathrm{meV}$ in EuTe and $300 \mathrm{meV}$ in EuSe. A level scheme, described by Guntherodt et al. ${ }^{6}$ is used to describe the absorption spectrum, showing good agreement with experiment. The position of the absorption line is tunable by the external magnetic field, due to the exchange interaction between electrons in the excited state and the localized spins in the crystal lattice. In EuTe, both lines show a quadratic dependence on the magnetic field intensity, as predicted theoretically for a transition tuned by the free-carrier exchange interaction. The quadratic dependence contrasts with the linear dependence seen in dilute magnetic 
semiconductors, ${ }^{12}$ and it can be traced to the fact that the spin of the photoexcited electron is parallel to the spin of the site where the excitation occurred, due to spin conservation in the optical transition. Faraday rotation in the transparency gap was also measured, showing a strongly nonlinear behavior, which is well explained by the changes in the absorption spectrum, as a function of the applied magnetic field, described above.

\section{EXPERIMENT}

EuTe and EuSe epitaxial layers were grown by molecular beam epitaxy (Riber 32P MBE) onto freshly cleaved (111) $\mathrm{BaF}_{2}$ substrates. X-ray and photoluminescence samples were used to characterize the EuTe samples. The crystalline quality was investigated by measuring the $\mathrm{x}$-ray rocking curve of the (222) Bragg reflection of the samples. The peak position corresponded to the bulk lattice parameter of $a_{0}=6.600 \mathrm{~A}$, and the full width at half maximum of the EuTe diffraction peak was about 400 arcsec, which is indicative of a good structural quality. PL measurements at $T=2 \mathrm{~K}$ were made, using a diode laser $(\lambda=403 \mathrm{~nm})$, and the sharp luminescence (FWHM $\sim 10 \mathrm{meV}$ ) at $1.922 \mathrm{eV}$ was detected, which is an indication of the high optical quality of the samples. ${ }^{8}$ The optical absorption spectra of the EuTe and EuSe were measured at $1.8 \mathrm{~K}$, using left and right circularly polarized light, in magnetic fields of intensity up to $10 \mathrm{~T}$. The sample (square shaped, of side length $2.5 \mathrm{~mm}$ ), was immersed in super fluid helium, and the light was conveyed to the sample, and collected from the sample, in the Faraday configuration, using optical fibers coupled to in situ miniature focusing optics and circular polarizers. Faraday rotation in the transparency gap was measured also using optical fibers to conduct and collect light, and in situ linear polarizers. Light went through a fixed linear polarizer just before entering the sample, and it went through a second linear polarizer after emerging from the sample. The second polarizer was rotated using a gear system, which was operated using a stepping motor, the minimum step being $0.0075^{\circ}$.

\section{RESULTS}

Figure 1 shows the absorption spectrum of a sample of EuTe, of thickness $0.18 \mu \mathrm{m}$, at $T=1.8 \mathrm{~K}$, for an applied magnetic field of 9.6 T. Under these conditions, the localized spins attain ferromagnetic order. The measured optical density increases linearly with the thickness of the epitaxial layer, ${ }^{11}$ demonstrating that the optical absorption is associated with electronic transitions in the bulk of the sample. For the Faraday geometry used here, the measured spectrum is strongly dependent on the direction of rotation of the circularly polarized light. The absorption of photons carrying an angular momentum of $+\hbar$, or $-\hbar$, in the magnetic field direction, are described by the spectra labeled by $\sigma^{+}$, or $\sigma^{-}$, respectively, in Fig. 1. In our previous investigation, ${ }^{5}$ whereby we studied thicker EuTe layers with a much greater optical density, the optical absorption spectrum could only be measured below a photon of $2.3 \mathrm{eV}$, and the $\sigma^{+}$line seen at about $2.35 \mathrm{eV}$ in Fig. 1 could not be measured.
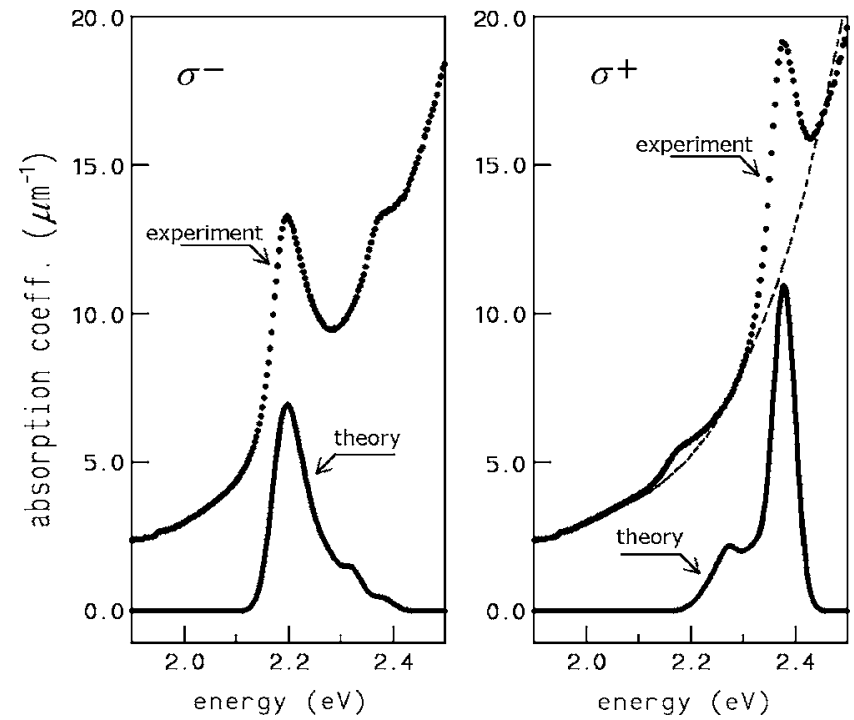

FIG. 1. Optical absorption in EuTe for $T=1.8 \mathrm{~K}$ and $B=9.6 \mathrm{~T}$. Dots represent experimental spectra and full lines the theoretical ones. The dashed curve in the right panel represents the absorption spectrum at $B=0$.

We used the theoretical model described in detail in Ref. 6 to generate a theoretical absorption line shape. In this model, the ground state consists of an $\mathrm{Eu}^{2+}$ in a $\left|4 f^{7}\left({ }^{8} S_{7 / 2}\right) M=-\frac{7}{2}\right\rangle$. The excited state is one of the electronic states within the manifold of levels $\left|4 f^{6}\left({ }^{7} F\right) J M_{J} ; X\right\rangle$, which consists of an $\mathrm{Eu}^{3+}$ core in a $4 f^{6}$ configuration, split by the spin-orbit interaction, described by a constant $\lambda_{4 f}$, and $X$ represents an electron in one of the six possible $5 d t_{2 g}$ orbitals, which compose the lower energy branch of the $5 d$ orbitals split by the crystal field of octahedral symmetry, ${ }^{6}$ whereby the spin-orbit interaction of the $5 d$ orbitals is described by a spin-orbit constant $\lambda_{5 d}$. The exchange interaction between the electron in the excited $d$ state and the localized spins is characterized by a constant $J_{d f} S .^{5}$ Because of the low temperatures used in the experiment, it is assumed that in the ground state only the $M=-\frac{7}{2}$ was populated (the minus sign arises because we define as positive the direction of the applied magnetic field).

The absorption coefficient at the photon energy $h \nu$, associated with transitions from the ground state to the excited ones (indexed by $f$ ), was calculated by

$$
\alpha(h \nu)=\sum_{f} \frac{\pi N h \nu e^{2}}{3 \epsilon_{0} \hbar c}\left|\boldsymbol{M}_{f}\right|^{2} g\left(h \nu-E_{f}\right)
$$

where $\boldsymbol{M}_{f}$ is the electric dipole matrix element between the initial state and a given final state, which can be calculated as described in Ref. $4, E_{f}$ is the energy of the excited state relative to the ground state, $N$ is the volume density of $\mathrm{Eu}$ atoms in the crystal, and $g(h \nu)$ is a normalized line-shape function, which we approximated by a Gaussian function. Figure 1 also shows the theoretical optical absorption spectrum, generated using Eq. (1). The parameters used to produce the theoretical spectrum were $N=1.4 \times 10^{28} \mathrm{~m}^{-3}$ (deduced from the lattice parameter $a_{0}$ for EuTe), $\lambda_{4 f}$ $=9.6 \mathrm{meV}, J_{d f} S=150 \mathrm{meV} .5$ The $4 f-5 d$ radial integral, $r_{f d}$ 
$=\int \mathcal{R}_{4 f} \mathcal{R}_{5 d} r^{3} d r$, which enters the dipole matrix element ${ }^{4} \boldsymbol{M}_{f}$, was taken to be $r_{f d}=0.18 \AA$. The model is quite insensitive to the value of parameter $\lambda_{5 d}$, as already noted by Pidgeon $e t$ al. ${ }^{13}$ All lines were assumed to be of full width at half maximum of $50 \mathrm{meV}$. Finally, the zero-field energy gap was fixed at $E_{G}(0)=2.327 \mathrm{eV}$ (see Fig. 4), where we define the gap as the lowest energy transition between the ground state and the manifold of excited states comprised in the electronic term $\left|4 f^{6}\left({ }^{7} F\right) J M_{J} ; X\right\rangle$. Good agreement between theory and experiment is found. The main difference between the theoretical and experimental spectra is the monotonously increasing absorption background, which is seen experimentally, due to the onset of absorption to higher energy levels, but which is absent in the theoretical spectrum, because these levels were not included in the model.

A qualitative understanding of the $\sigma^{+}$and $\sigma^{-}$absorption spectra can be attained quite simply in terms of the energy level scheme we used, if we ignore the spin-orbit interaction associated with the excited $5 d$ electron (i.e., putting $\lambda_{5 d}=0$ ), which plays only a minor role as discussed above. The spinorbit interaction within the $\mathrm{Eu}^{3+}$ core leads to the formation of seven levels $4 f^{6}\left(F_{J}\right)(J=0, \ldots, 6)$, spaced in energy by the Landé interval rule. The total excitation energy, relative to the ground $\left[4 f^{7}\left(S_{7 / 2}\right)\right]$ state, will be given the energy of the $4 f^{6}\left(F_{J}\right)$ state, plus the energy of an electron in one of the three degenerate $5 d\left(t_{2 g}\right)$ orbitals $\left(d_{x y} d_{y z}\right.$, and $\left.d_{z x}\right)$. Theoretically, it is found that the oscillator strength for transitions between the ground state and states of low values of $J$ is large for $\sigma^{-}$polarization, and small for $\sigma^{+}$polarization, whereas the reverse is true for large values of $J$. Due to the level broadening, the overall spectrum is described by a strong $\sigma^{-}$line as seen in the low energy side of the absorption spectrum, and a strong $\sigma^{+}$line on its high energy side. The $\sigma^{-}$line is approximately centered midway between the $\left|4 f^{7}\left({ }^{8} S_{7 / 2}\right) M=-\frac{7}{2}\right\rangle \rightarrow\left|4 f^{6}\left({ }^{7} F\right) J M_{J} ; X\right\rangle$ for $J=1$ and $J=2$, whereas the $\sigma^{+}$line is approximately centered around the $\left|4 f^{7}\left({ }^{8} S_{7 / 2}\right) M=-\frac{7}{2}\right\rangle \rightarrow\left|4 f^{6}\left({ }^{7} F\right) J M_{J} ; X\right\rangle$ for $J^{\prime}=6$, and hence the $\sigma^{-}$and $\sigma^{+}$lines are separated by $\Delta E=\frac{1}{2} \lambda_{4 f}\left[J^{\prime}\left(J^{\prime}+1\right)\right.$ $-J(J+1)] \approx 19 \lambda_{4 f}$.

Figure 2 shows the experimental and theoretical absorption spectra for the EuSe sample, of thickness $0.34 \mu \mathrm{m}$, at $B=3.0 \mathrm{~T}$. At the temperature used $(T=1.8 \mathrm{~K})$, the order of the spins becomes ferromagnetic above $B=2 \mathrm{~T} .{ }^{14}$ The same theoretical model used for EuTe also describes well the absorption spectrum of EuSe if the following parameters are used: $N=1.7 \times 10^{28} \mathrm{~m}^{-3}$ (deduced from the lattice parameter $a_{0}=6.195 \AA$ for EuSe), ${ }^{14} \lambda_{4 f}=0.014 \mathrm{eV}, J_{d f} S=0.104 \mathrm{eV}$ (taken from the shift of the absorption line between $B=0$ and the high field limit, when the ferromagnetic arrangement is achieved $^{5}$ ), and $E_{G}(0)=2.092 \mathrm{eV}$. The energy levels were broadened by a Gaussian of FWHM $=80 \mathrm{meV}$. The $4 f-5 d$ radial integral was fixed at $r_{f d}=0.24 \AA$, which is in good agreement with the estimate given in Ref. 4 . The absorption spectra for EuSe measured here can be compared to the reflectivity spectrum reported in Ref. 13, and it is found that the maxima seen in the absorption have a near coincidence with the minima in the reflectivity spectra reported in Ref. 13.

Next we will analyze the change in the peak positions in the absorption spectra as a function of the external field in
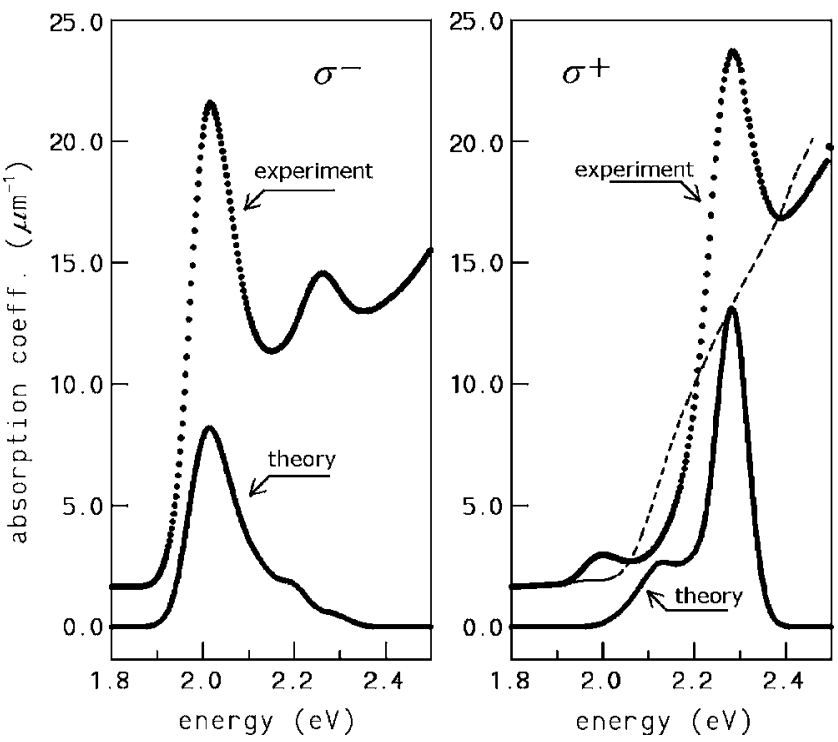

FIG. 2. Optical absorption in EuSe for $T=1.8 \mathrm{~K}$ and $B=3.0 \mathrm{~T}$. Dots represent experimental spectra and full lines the theoretical ones. The dashed curve in the right panel represents the absorption spectrum at $B=0$.

EuTe. The absorption coefficient decreases when the magnetic field decreases, however, the position of the weak absorption maximum could still be picked up in the absorption spectrum down to zero field. To reduce the effect of the background monotonous absorption spectrum, and to pick the absorption maxima with greater precision, the zero field absorption spectrum was subtracted from the spectra measured at nonzero fields. The magnetic field-induced optical absorption measured in this way is shown in Fig. 3 for a number of magnetic field values. The strong reduction in the absorption can be clearly seen. Figure 4 shows the position
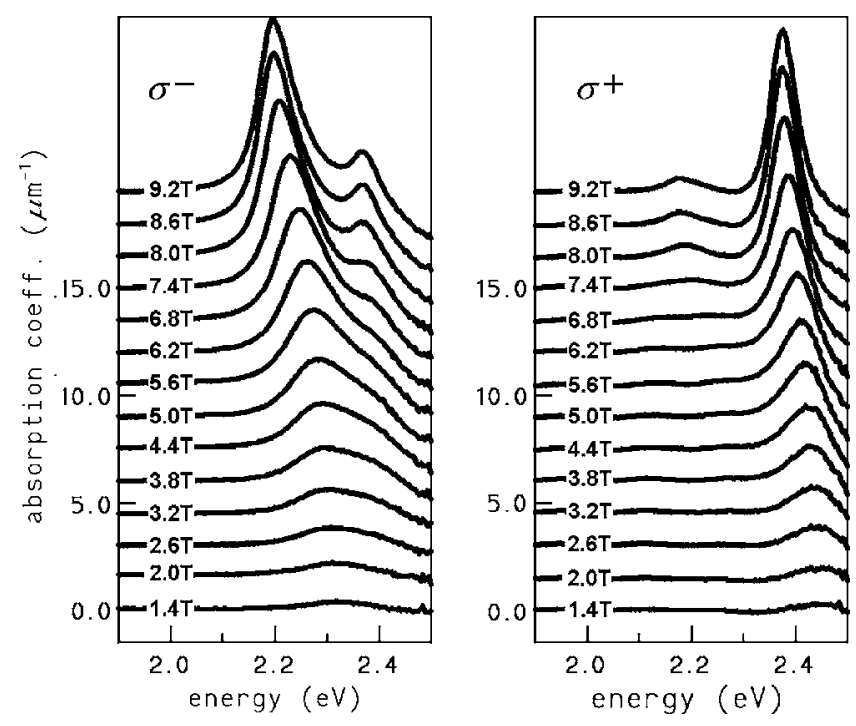

FIG. 3. Magnetic field induced polarized optical absorption in EuTe. The zero-field absortion spectrum was subtracted from each curve shown. For clarity, successive absorption spectra were displaced vertically by $1.5 \mu \mathrm{m}^{-1}$ from the previous one. The magnetic field associated with each curve is indicated. 


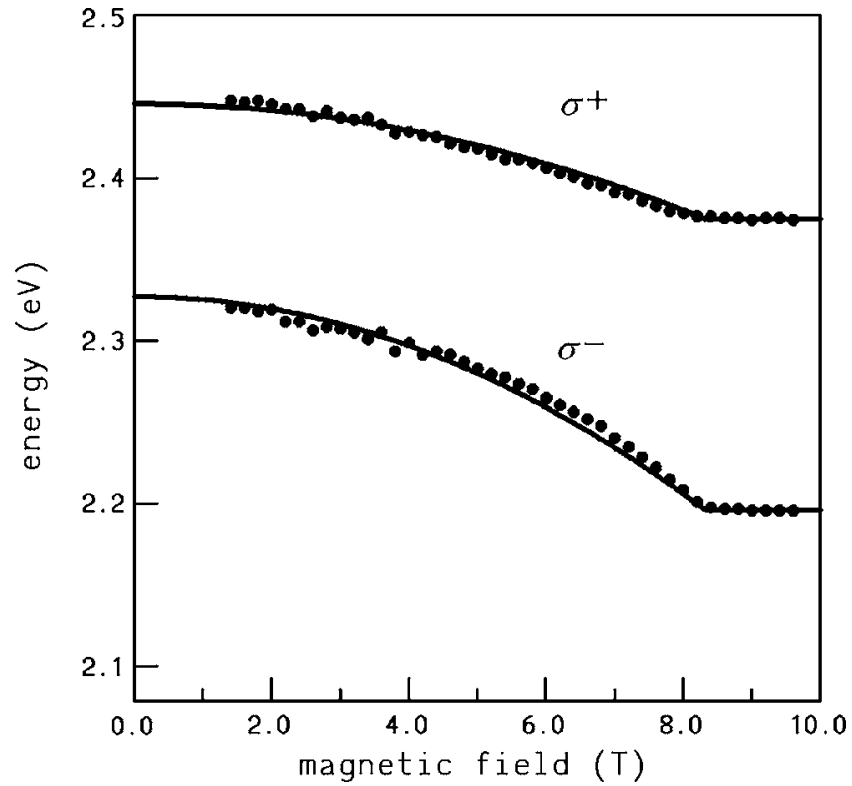

FIG. 4. Peak position of the maxima seen in the polarized absorption spectra in EuTe as a function of magnetic field. Dots are the measured values; full lines represent the theoretical dependence.

of the absorption lines as a function of magnetic field, obtained from Fig. 3. The change in the band gap is clearly nonlinear. Solid lines in Fig. 4 represent a fit of the dots shown using the theory described in Ref. 5, which predicts a quadratic dependence of the line position on magnetic field intensity, until complete ferromagnetic order is attained

$$
E_{G}(B)=E_{G}(0)-J_{d f} S \times \begin{cases}\left(\frac{B_{\text {int }}}{B_{\text {Sat }}}\right)^{2} & \text { if } B_{\text {int }}<B_{\text {Sat }} \\ 1 & \text { if } B_{\text {int }}>B_{\text {Sat }},\end{cases}
$$

where $B_{\text {Sat }}=7.22 \mathrm{~T}$ is the saturation field for EuTe, and $B_{\text {int }}(T)=B-1.1, B$ is the external magnetic field. The best fit $J_{d f} S$ values are different for the $\sigma^{+}$and $\sigma^{-}$lines. For the $\sigma^{+}$ line, we find $J_{d f} S=0.13 \mathrm{eV}$, in agreement with measurements done on thicker samples. ${ }^{5}$ The position of the $\sigma^{+}$line was measured for the first time, and the best fit value is $J_{d f} S$ $=0.09 \mathrm{eV}$.

For a given electronic state, the exchange constant, $J_{d f} S$, will be determined by the overlap of the extended $\left[5 d\left(t_{2 g}\right)\right]$ electronic wave function and the electronic wave function $\left[4 f^{7}\left(S_{7 / 2}\right)\right]$, localized at the $\mathrm{Eu}^{2+}$ sites in the crystal. The difference in the exchange constants, $J_{d \rho} S$, for the $\sigma^{+}$and $\sigma^{-}$ lines, is an indication that in the excited electronic wave functions, associated with the $\sigma^{+}$and $\sigma^{-}$lines, have a different overlap with the localized spins. This contradicts our simple level scheme (see the previous discussion for the limit when $\lambda_{5 d}=0$ ), whereby all three $5 d$ electronic states are equivalent. Nevertheless, the assumption that $J_{d f} S$ is constant for all lines can still provide an approximate description of the change in the peak positions of the absorption lines when $B$ is increased from zero, as shown in Fig. 4.

The large increase in the absorption coefficient for the $\sigma^{+}$and $\sigma^{-}$lines, when the magnetic field increases, indicates that the oscillator strength for the

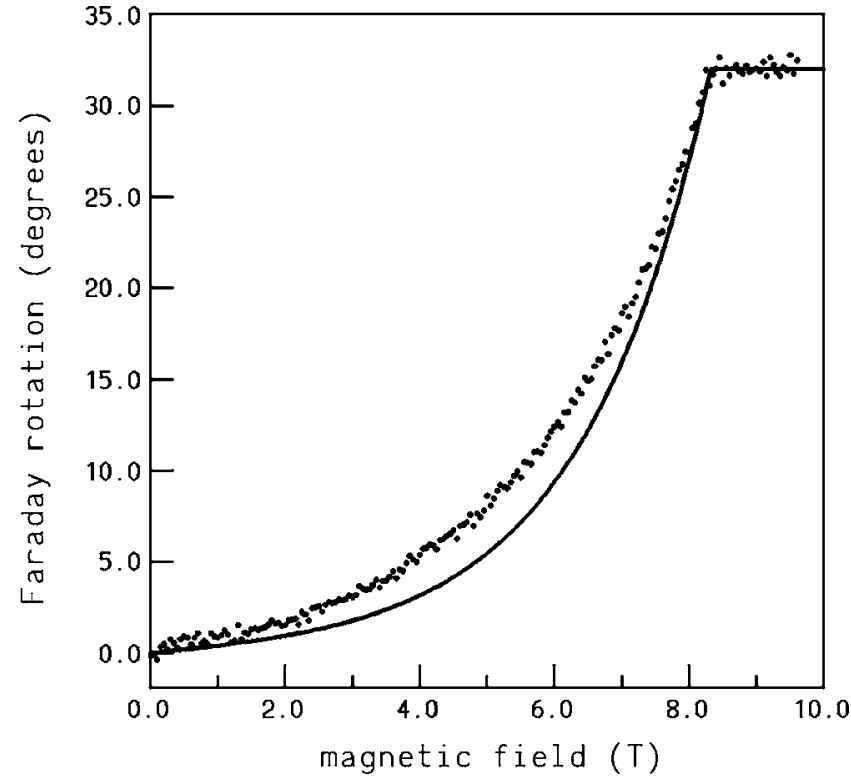

FIG. 5. Faraday rotation for a photon energy of $h \nu=1.934 \mathrm{eV}$ in EuTe of thickness $1.75 \mu \mathrm{m}$. Dots represent experimental data and the full line represents theory.

$4 f^{7}\left({ }^{8} S_{7 / 2}\right) \rightarrow 4 f^{6}\left(F_{J}\right) 5 d\left(t_{2 g}\right)$ optical transitions increases with the magnetic field intensity. An increase in the oscillator strength of an optical transition associated with an exciton creation, when the applied magnetic field increases, is a very well known fact, for instance, in III-V semiconductors, and it is well explained by an increasing compression of the electron-hole exciton wave function when the magnetic field increases. ${ }^{15}$ By the same reasoning, we expect that the magnetic field should induce a similar compression of electrons and holes to the one seen in III-V semiconductors. The large change in absorption coefficients for the $\sigma^{+}$and $\sigma^{-}$lines, when the magnetic field increases, are then to be associated with a compression of the $5 d\left(t_{2 g}\right)$ electron around the $4 f^{6}\left(F_{J}\right)$ hole state. A quantitative analysis for the magnetic field dependence would require modeling of the wave functions using, for example, the variational principle, as in Ref. 15 , and is outside the scope of the present investigation. We did not do the same analysis of the changes in the absorption spectrum with the magnetic field intensity for the EuSe sample, because the phase diagram for EuSe is much more complicated than for EuTe, ${ }^{14}$ therefore the absorption peak positions will not follow the simple magnetic field dependence described by Eq. (2).

Finally, Fig. 5 shows the Faraday rotation measured for a EuTe of thickness $1.75 \mu \mathrm{m}$, in the transparency gap, using the laser light of energy $h \nu=1.934 \mathrm{eV}$. A strongly nonlinear behavior is seen at low fields, in contrast to previous reports (see, for instance, Ref. 17). This nonlinearity is directly related to the changes in the optical absorption described above. A simple approximation to describe the Faraday rotation angle below saturation is ${ }^{18}$

$$
\Phi_{F}(B)=\frac{\alpha(B)}{\alpha_{s}} \frac{E_{G s}^{2}-(h \nu)^{2}}{E_{G}^{2}(B)-(h \nu)^{2}} \frac{M(B)}{M_{s}} \Phi_{F s},
$$

where $\alpha(B), E_{G}(B), M(B)$ represent the absorption coefficient, the energy band gap, and the magnetization of the 
sample, respectively, at a field $B$ below saturation, $\alpha_{s}, E_{G s}$, and $M_{s}$ are their saturation values, and $\Phi_{F s}$ is the saturation value of the Faraday rotation angle. Taking the magnetization dependence below saturation as a linear function of $B$, as described in Ref. 5, the absorption coefficient as equal to the peak value of the $\sigma^{-}$absorption line seen in Fig. 3, and the band-gap dependence on $B$ as equal to the energy position of the peak of the $\sigma^{-}$absorption line (Fig. 4), the theoretical dependence of $\Phi_{F}(B)$ can be traced using Eq. (3), and it is shown in Fig. 5. The good qualitative agreement with the experimental values, without using any adjustable parameters, indicates that the nonlinearity in the Faraday rotation angle can be attributed to a narrowing of the band gap, and a concomitant increase in the oscillator strength of the bandedge absorption, when the applied magnetic field increases.

\section{CONCLUSION}

The optical absorption spectra of thin EuTe and EuSe epitaxial layers grown by MBE were investigated, in high magnetic fields, when the arrangement of the localized spins in the crystal lattice is ferromagnetic. A doublet of lines is observed, which can be separated from one another by using circularly polarized light in opposite directions. The experimental results can be explained very well on the basis of a model in which the excited state is built from a narrow band of $d$ character. The oscillator strength of the absorption shows a strong dependence on the intensity of the applied magnetic field. This suggests that the wave function of the extended state could be changing from a Bloch-like extended state towards a localized state when the magnetic field increases. In EuTe, the absorption lines display a quadratic dependence on external magnetic field, which is explained by spin conservation in the optical electronic transition. The Faraday rotation is strongly nonlinear below the saturation field, which is well explained by the changes in the absorption spectra when the applied magnetic field increases.

\section{ACKNOWLEDGMENTS}

The authors gratefully acknowledge the support provided by CNPq (Project No. 308116/2004-6) and FAPESP (Project No. 04/08415-6).
${ }^{1}$ S. A. Wolf, D. D. Awschalom, R. A. Buhrman, J. M. Daughton, S. von Molnár, M. L. Roukes, A. Y. Chtchelkanova, and D. M. Treger, Science 294, 1488 (2001).

${ }^{2}$ R. T. Lechner, G. Springholz, T. U. Schülli, J. Stangl, T. Schwarzl, and G. Bauer, Phys. Rev. Lett. 94, 157201 (2005).

${ }^{3}$ T. Kasuya, J. Magn. Magn. Mater. 195, 141 (1999).

${ }^{4}$ M. J. Freiser, S. Methfessel, and F. Holtzberg, J. Appl. Phys. 39, 900 (1968).

${ }^{5}$ A. B. Henriques, L. K. Hanamoto, N. F. Oliveira, Jr., P. Rappl, E. Abramoff, and Y. Ueta, J. Phys.: Condens. Matter 16, 5597 (2004).

${ }^{6}$ G. Güntherodt, P. Wachter, and D. M. Imboden, Phys. Kondens. Mater. 12, 292 (1971).

${ }^{7}$ G. Güntherodt, Phys. Cond. Matter. 18, 37 (1974).

${ }^{8}$ W. Heiss, G. Prechtl, and G. Springholz, Appl. Phys. Lett. 78, 3484 (2001).

${ }^{9}$ W. Heiss, G. Prechtl, and G. Springholz, Phys. Rev. B 63, 165323 (2001).

${ }^{10}$ W. Heiss, R. Kirchschlager, G. Springholz, Z. Chen, M. Debnath, and Y. Oka, Phys. Rev. B 70, 035209 (2004).

${ }^{11}$ A. B. Henriques, L. K. Hanamoto, E. Abramof, A. Y. Ueta, and P. H. O. Rappl, in Proceedings of the 27th International Conference on the Physics of Semiconductors, edited by José Menéndez and Chris G. Van de Walle (AIP, New York, 2004), Vol. 772, p. 1019.

${ }^{12}$ J. K. Furdyna, J. Appl. Phys. 64, R29 (1988).

${ }^{13}$ C. R. Pidgeon, J. Feinleib, W. J. Scouler, J. Hanus, J. O. Dimmock, and T. B. Reed, Solid State Commun. 7, 1323 (1969).

${ }^{14} \mathrm{P}$. Wachter, Handbook on the Physics and Chemistry of Rare Earths (North-Holland, Amsterdam, 1979), Vol. 1, p. 507.

${ }^{15}$ Mitsuru Sugawara, Phys. Rev. B 45, R11423 (1992).

${ }^{16}$ R. Akimoto, M. Kobayashi, and T. Suzuki, J. Phys.: Condens. Matter 8, 105 (1996).

${ }^{17}$ H. Hori, R. Akimoto, M. Kobayashi, S. Miyamoto, M. Furusawa, N. M. Kreines, A. Yamagishi, and M. Date, Physica B 201, 438 (1994).

${ }^{18}$ N. Bloembergen, P. S. Pershan, and L. R. Wilcox, Phys. Rev. 120, 2014 (1960). 